\title{
Numerical study of laser line thermography for crack detection at high temperature
}

\author{
by Nithin Puthiyaveettil1, Renil Kidangan1, Sreedhar Unnikrishnakurup2, C V Krishnamurthy1, \\ Mathias Zeigler2, Philipp Myrach2 and Krishnan Balasubramaniam1
}

1 Centre for Non-Destructive Evaluation, Mechanical Engineering Department, IIT Madras, India

2 BAM Federal Institute for Materials Research and Testing, 12205 Berlin, Germany

nithinvengara@gmail.com

\begin{abstract}
The detection of cracks before the failure is highly significant when it comes to safety-relevant structures. Crack detection in metallic samples at high surface temperature is one of the challenging situation in manufacturing industries. Laser thermography has already proved its detection capability of surface cracks in metallic samples at room temperature. In this work a continuous wave (CW) laser use to generate a laser, which is using to scan the metal surface with notch. The corresponding heat distribution on the surface monitored using infrared thermal (IR) camera. A simplified 3D model for laser thermography is developed and validated with experimental results. A dedicated image processing algorithm developed to improve the detectability of the cracks. To understand the dependency of surface temperature, laser power, laser scanning speed etc. in defect detection, we carried out parametric studies with our validated model. Here we report the capability of laser thermography in crack detection at elevated temperature.
\end{abstract}

Keywords: Thermal contrast, Laser Thermography, FEM, Elevated temperature, Surface cracks

\section{Work details}

The commercial Finite Element (FE) package was used for the numerical modelling of heat transfer phenomena during a laser line heating. The model was developed using rectangular block of steel having a $100 \mathrm{~mm}$ length, $100 \mathrm{~mm}$ width and $40 \mathrm{~mm}$ thickness. The objective of this work is to develop numerical models which can address the effect of a laser line heating on a sample. When the defect introduced in the model the heat transfer phenomenon is altered. This phenomenon is handled as an obstruction for the heat flow in the numerical mode Influence of parameter on thermal contrast studied.

Fig.1 show the increase in maximum surface temperature with different laser power is shown. Both experimental and modeling results are shown in the plot. Both results are showing compromising results. When the laser power increases, increase in surface temperature is linearly increasing. Temperature dependency on process parameter are studied.

- Thermal contrast is decreasing with increasing surface temperature

- Thermal contrast is increasing with increasing laser power

- Thermal contrast is decreasing with increasing scanning speed

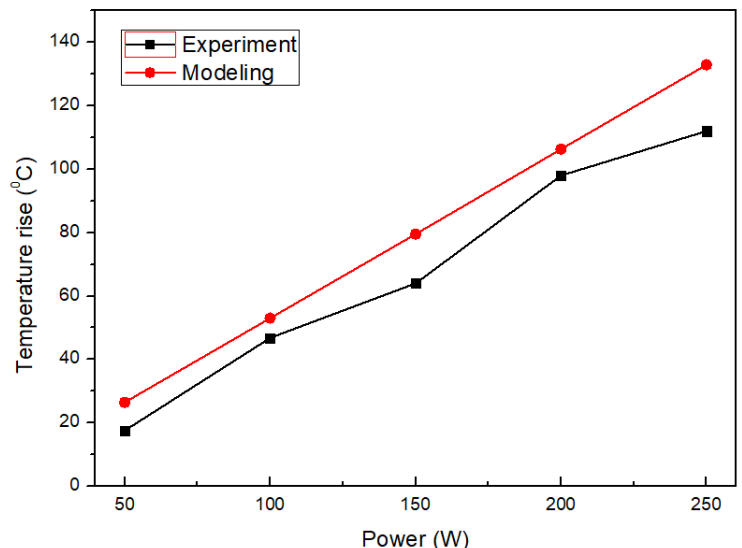

Fig.1 Increase in maximum surface temperature with different laser power

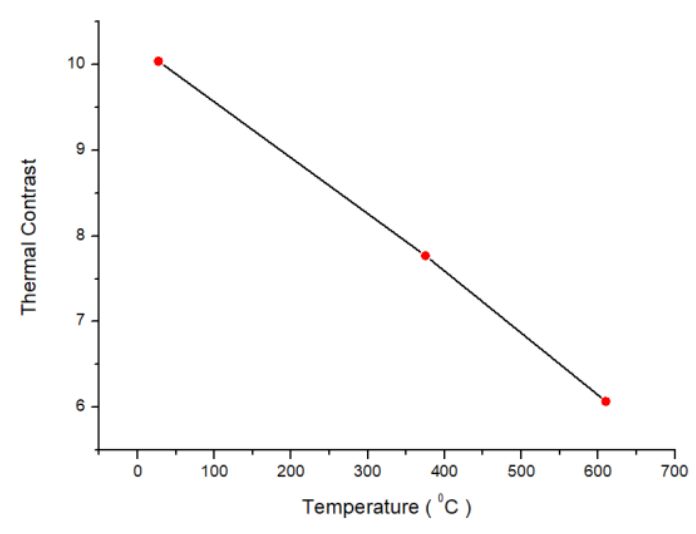

Fig.2 Thermal contrast Vs surface temperature 


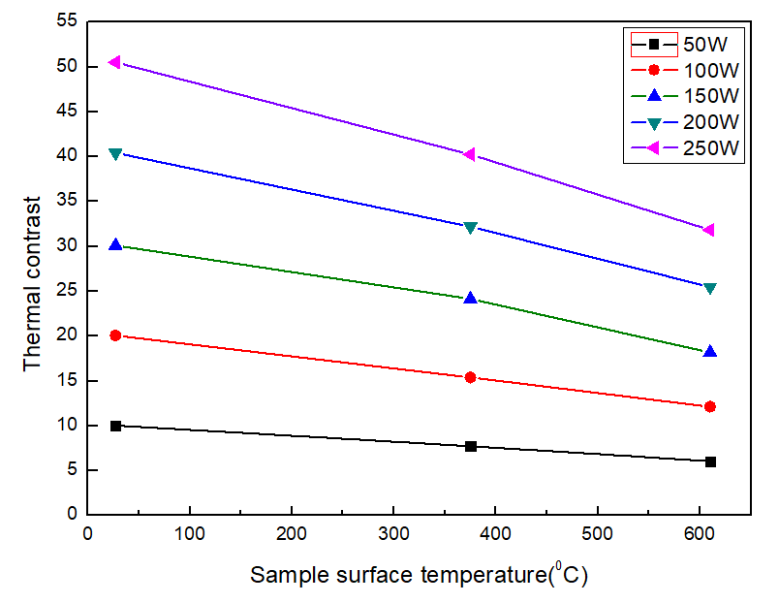

Fig.3 Influence of scanning laser power on thermal contrast

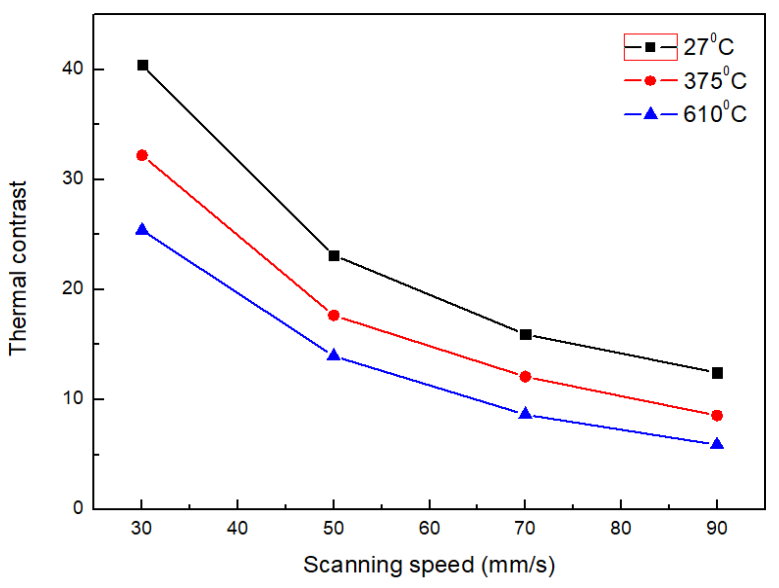

Fig.4 Influence of laser scanning speed on thermal contrast.

\section{Acknowledgements:}

This work is financially supported from Indo-German Science and Technological Centre (IGSTC) under the project entitled Advanced Manufacturing Process Monitoring using in-line LASer Thermography (AMPLAST).

\section{REFERENCES}

[1] S. E. Burrows, A. Rashed, D. P. Almond, S. Dixon, "Combined laser spot imaging thermography and ultrasonic measurementsfor crack detection," NDT \&E Intern. (Special Issue: Thermographic NDT) 22(2-3)(2007).

[2] Philipp Myrach, Mathias Ziegler, Christiane Maierhofer, Marc Kreutzbruck "Influence of the Acquisition Parameters on the Performance of Laser-Thermography for Crack Detection in Metallic Components", proc. QNDE2013(2013)

[3] Schlichting J., Maierhofer C., Kreutzbruck M., "Defect sizing by local excitation thermography", QIRT Journal 8(1), 51 (2011).

[4] T. Li, D. P. Almond, D. Andrew, S. Rees, B. Weekes, "Crack imaging by scanning pulsed laser spot thermography", NDT\&E Intern. 44, 216(2011)

[5] J. Schlichting, M. Ziegler, C. Maierhofer, M. Kreutzbruck, "Efficient data evaluation for thermographic crack detection", QIRT Journal, Short Communications 8(1), 119(2011).

[6] Nithin P V, Sruthikrishna K P, Renil Thomas, Sreedhar Unnikrishnakurup, C V Krishnamurthy, Mathias Zeigler, Philippe Myrach, Krishnan Balasubramaniam, "In-line laser thermography for crack detection at elevated temperature: A Numerical modeling study”, proc QIRT 2016 